\title{
The Potential of the Maximum Cross-Correlation Technique to Estimate Surface Currents From Thermal AVHRR Global Area Coverage Data
}

\author{
Steffen Dransfeld, Gilles Larnicol, and Pierre-Yves Le Traon
}

\begin{abstract}
Having already shown its potential of deriving the vector fields representing the ocean-surface advection from sequential 1.1-km-resolution local area coverage (LAC) Advanced Very High Resolution Radiometer (AVHRR) images, the maximum cross-correlation (MCC) technique here is applied to four 4.4-km-resolution global area coverage (GAC) AVHRR images. The resulting three vector fields are compared to the vector fields obtained from the LAC imagery corresponding to the same satellite passages. To quantify the reduction in accuracy inevitable when applying the method to the lower resolution imagery, the LAC vector fields were assumed to be error free. The deviation of the GAC vectors from the $\mathrm{LAC}$ vectors is expressed as percentage errors of the signal variance of meridional $u$ and zonal $v$ velocity components, and they are $16 \% / 30 \%$, respectively, for the best case and $62 \% / 117 \%$ and $92 \% / 111 \%$ for the other two cases. These results indicate that, in its present state, the GAC data do not allow the MCC technique to extract reliable current-vector information from it.
\end{abstract}

Index Terms-Image motion, infrared imaging, marine technology, remote sensing.

\section{INTRODUCTION}

$\mathbf{T}$ HE FREQUENT global coverage of the National Oceanic and Atmospheric Administration's (NOAA) polar orbiters carrying the Advanced Very High Resolution Radiometer (AVHRR) instrument regularly provides the ocean science community with the thermal state of the ocean surface. For many years now this information has also been used to observe and quantify surface advective motion. The capacity of the instrument to fly over the same spot within relatively short time scales allows the observation and tracking of the same thermal features between satellite passes. Various feature-tracking and pattern-recognition techniques have been applied to follow the propagation of these features [1], [2], [4], [6], in which the maximum cross-correlation (MCC) method has singled itself out as a convenient and robust method for a routine processing of AVHRR local area coverage (LAC) imagery. The most comprehensive recent study of the method has been carried out by Bowen et al. [1] who analyzed a seven-year archive of LAC

Manuscript received December 11, 2005; revised April 11, 2006.

S. Dransfeld is with the Institut für Meereskunde, University of Hamburg, 20146 Hamburg, Germany (e-mail: dransfeld@ifm.uni-hamburg.de).

G. Larnicol is with the Collect Localisation Satellite, Direction d'Océanographie Spatiale, 31526 Ramonville St Agne, France.

P.-Y. Le Traon is with the IFREMER Centre de Brest, 29280 Plouzané, France.

Digital Object Identifier 10.1109/LGRS.2006.878439

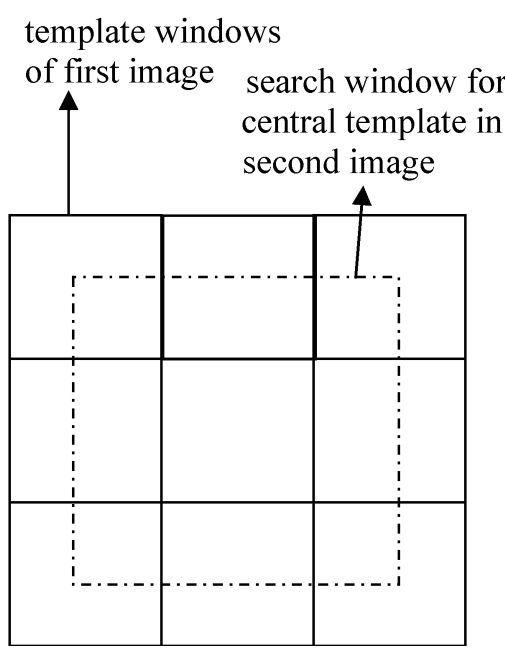

Fig. 1. Division of the first image into templates (solid lines) and search area (dashed line) corresponding to the central template.

imagery from the east Australian current system and estimated a method precision between 0.08 and $0.2 \mathrm{~m} / \mathrm{s} \mathrm{rms}$.

While the current estimates derived from the LAC images may be accurate, they are generally limited to regional scales caused by the high resolution of the images. The offshore and shipping industries, however, are more concerned about larger scale current products, in which global area coverage (GAC) images are a more appropriate dataset in terms of developing an operational current product. In this letter, we have thus attempted to quantify the loss in accuracy resulting from the application of the MCC technique to GAC images as opposed to the corresponding LAC images.

\section{MCC TeChNique Description}

The method is based on identifying a maximum cross correlation between the features of the individual subscenes of the two sequential thermal images. For a full description see [5]. The first image is divided into a number of template tiles (see Fig. 1) whose size depends on the oceanic flow structures that need to be resolved. Each template window will be searched for in the second image by using a search window (dashed line in Figs. 1 and 2), whose size depends on the maximum current speed that is expected between two sequential images. Because the search window is much larger than its corresponding template window, the neighboring search windows overlap. 


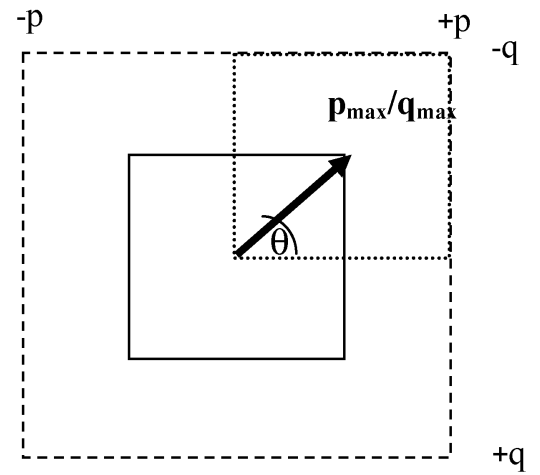

Fig. 2. Vector indicating where in the second image the center of the window (dotted line) closest to the original template (solid line) lies.

The pixel values of the template window are $A(x, y)$, and the corresponding pixels in the second window are $B(x, y)$ at no lag and $B(x+p, y+q)$ for a lag $(p, q)$. The normalized spatial cross-correlation function between the template and the search window at lag $(p, q)$ is defined as

$$
\begin{aligned}
r(p, q)= & \frac{1}{\sigma_{A} \sigma_{B}^{l}} \\
& \times \sum \sum\{[A(x, y)-\bar{A}(x, y)] \\
& \cdot[B(x+p, y+q)-\bar{B}(x+p, y+q)]\} \\
= & \frac{\operatorname{Cov}(p, q)}{\sigma_{A} \sigma_{B}^{l}} .
\end{aligned}
$$

The summation is performed over all the $x$ and $y$ values of the template window. $\bar{A}(x, y)$ and $\bar{B}(x+p, y+q)$ correspond to the mean values of each window. $\operatorname{Cov}(p, q)$ is the covariance of $A$ and $B$ at lag $(p, q)$, and $\sigma_{A}$ and $\sigma_{B}$ are the standard deviations of the template and lagged window entering the correlation calculation. The displacement $(p, q)$ at the maximum value of the cross correlation determines the advective velocity $c$, as given by the following:

$$
\begin{aligned}
& c=\frac{\left[\left(p_{\max } \Delta x\right)^{2}+\left(q_{\max } \Delta y\right)^{2}\right]^{1 / 2}}{\Delta t} \\
& \theta=\arctan \left(q_{\max } \Delta y / p_{\max } \Delta x\right) .
\end{aligned}
$$

$\Delta x$ and $\Delta y$ represent the spatial interval and the pixel size, respectively, $p$ max and $q$ max represent the lag values for the MCC, and $\Delta t$ represents the time interval between both images. The direction of the motion of the displacement is given by $\theta$, as shown in (3).

\section{Preprocessing Performed on Images}

To be able to apply the MCC technique to the AVHRR LAC and GAC images, these had to undergo a reprojection of the satellite swath radiances onto a geographic grid and a correction of the attitude errors, which minimized geographical offsets between the successive images. Any offsets may introduce spurious current vectors that are not caused by natural advections. To correct for attitude errors, an algorithm developed at the Colorado Center for Astrodynamical Research (CCAR) was
TABLE I

ACQUisition Time AND SATELlite For EACH IMAGE

\begin{tabular}{|l|l|l|l|l|}
\hline Satellite & NOAA17 & NOAA16 & NOAA17 & NOAA16 \\
\hline Date/Time & $07 / 08 / 04$ & $07 / 08 / 04$ & $08 / 08 / 04$ & $08 / 08 / 04$ \\
(UTC) & $18: 43: 39$ & $22: 05: 55$ & $06: 04: 23$ & $10: 30: 30$ \\
\hline
\end{tabular}

used that geolocates the images to an estimated accuracy of about $1 \mathrm{~km}$ for all the coastal oceans. The images used in this letter are from four sequential satellite passes of a part of the Californian coastline. For each passage, we have the corresponding LAC and GAC image, thus allowing us to apply the MCC method to a sequence of LAC and GAC images that are from the same instant in time and the same area. Table I shows the acquisition time and satellite for each LAC/GAC image pair. The images were obtained from NOAA's Satellite Active Archive.

Rather than using the sea-surface temperature values that are based on the radiances of several channels of the AVHRR instrument and thus combine the noise contained in each channel, channel $4(10.6 \mu \mathrm{m})$ brightness temperatures, only containing the noise of that channel, were used. Both the GAC and LAC images consist of $512 \times 512$ pixels and have a resolution of $1.1 \mathrm{~km}$. The images were sampled in a $1.1-\mathrm{km}$ grid using an indirect navigation algorithm that combines a geographical grid with an orbital model to associate a scan element (line-spot value) from the field of vision with the correct pixel in the geographical grid. The algorithm computes a line-spot value for every eighth pixel in both the lat/long directions, and bilinear interpolation is used to calculate the remaining line-spot values that are used to index the field of vision and retrieve the data values. All images were cloud filtered, and cloudy as well as land-masked pixels were set to zero. According to Emery et al. [3], the correlations were computed for the windows containing at least $60 \%$ of the pixels with a brightness temperature value, to maintain the statistical significance of the correlations.

\section{Result AND Discussion}

In order to quantify the degradation of the MCC GAC vector fields relative to the LAC fields, the errors of each $u$ and $v$ velocity components ( $u=$ meridional velocity and $v=$ zonal velocity) were expressed as percentages of LAC signal variances. The following expressions explain how these are computed:

$$
\begin{aligned}
u_{L} \mathrm{rms} & =\sqrt{\frac{1}{n} \sum_{k=1}^{n}\left[u_{L}^{2}\right]} \\
u_{L}-u_{G} \mathrm{rms} & =\sqrt{\frac{1}{n} \sum_{k=1}^{n}\left(u_{L}-u_{G}\right)^{2}} \\
\% \text { error of signal variance } & =\left(\frac{u_{L}-u_{G} \mathrm{rms}}{u_{L} \mathrm{rms}}\right)^{2}
\end{aligned}
$$

where the subscripts $L$ and $G$ correspond to the LAC and GAC values, respectively, and $n$ is the number of vectors originating at identical coordinates. Errors were calculated for 


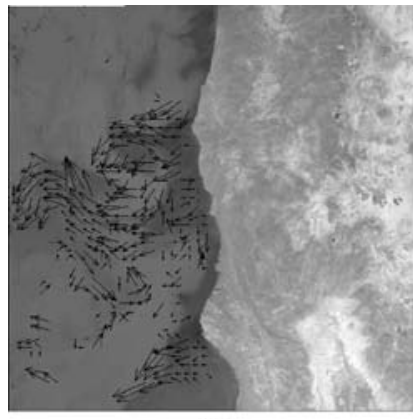

(a)

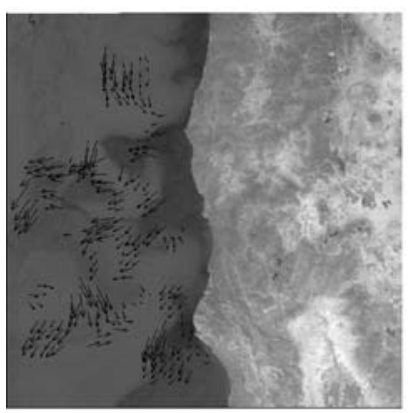

(c)

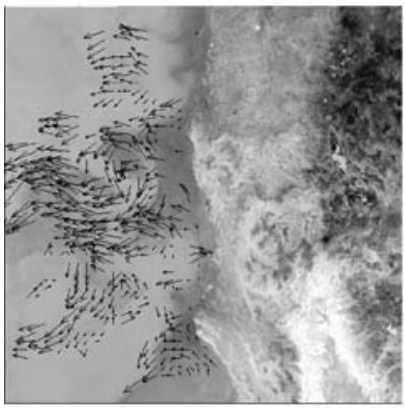

(e)

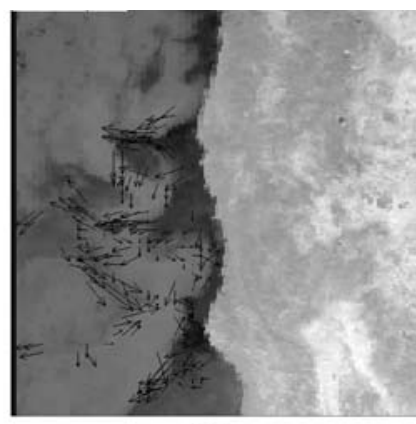

(b)

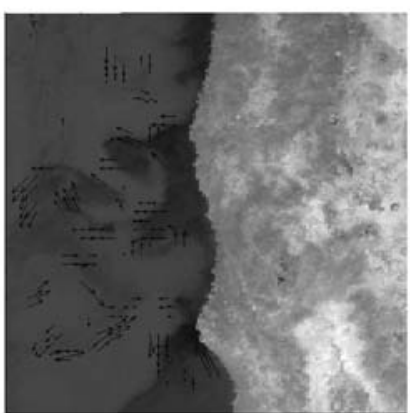

(d)

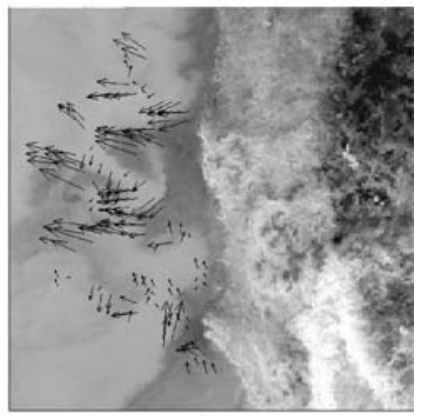

(f)
Fig. 3. (a) LAC vector field 18:43-22:05. (b) GAC vector field 18:43-22:05 (c) LAC vector field 22:05-06:04. (d) GAC vector field 22:05-06:04. (e) LAC vector field 06:04-10:30. (f) GAC vector field 06:04-10:30.

both vector magnitudes and directions. Fig. 3(a)-(f) shows the LAC and GAC vector fields obtained by applying the MCC algorithm to the image sequences. The parameters specific to the MCC are a 22-pixel initial template box, and the range that this template is searched for in the second image is also 22 pixels. This means that there is a 22-pixel zone all around the template box, where the template is searched for. After each search, the template box is moved 11 pixels further along, so that a vector is calculated every 11 pixels. A filter was applied to remove the vectors that correspond to a low cross correlation (lower than 0.75 ), and only the vectors that have three or more neighboring vectors, indicating similar feature propagations within two pixels in both directions, were kept (a vector based on a 15-pixel propagation was only kept if at least three vectors around it showed propagations of 13-17 pixels). This assured a spatial homogeneity of the vector field. Some vectors appear to be alone, as they have neighboring vectors that do not survive the filtering restrictions.

Table II shows the $u$ and $v$ error statistics of the GAC vectors relative to the LAC vectors obtained from expressions (4)-(6).
TABLE II

ERROR STATISTICS OF THE $u$ AND $v$ GAC VALUES RELATIVE TO THE LAC VALUES

\begin{tabular}{|l|c|c|c|c|}
\hline $\begin{array}{l}\text { Component and } \\
\text { number of vectors } \\
\text { entering statistics }\end{array}$ & LAC rms & GAC rms & LAC-GAC rms & $\begin{array}{c}\text { LAC-GAC rms } \\
\text { as of LAC } \\
\text { signal variance }\end{array}$ \\
\hline \multicolumn{5}{|c|}{ Image pair 18:43-22:05 } \\
\hline $\mathbf{U}(\mathbf{c m} / \mathbf{s})(\mathbf{1 1 5})$ & 28.72 & 37.89 & 22.73 & 62.65 \\
\hline V (cm/s) (111) & 20.23 & 28.18 & 21.85 & 116.60 \\
\hline \multicolumn{5}{|c|}{ Image pair 22:05-06:04 } \\
\hline $\mathbf{U}(\mathbf{c m} / \mathbf{s})(\mathbf{6 4})$ & 22.28 & 21.77 & 9.05 & 16.48 \\
\hline V (cm/s) (65) & 27.97 & 20.72 & 15.43 & 30.43 \\
\hline \multicolumn{5}{|c|}{ Image pair 06:04-10:30 } \\
\hline $\mathbf{U}(\mathbf{c m} / \mathbf{s})(\mathbf{1 1 0})$ & 29.93 & 42.13 & 28.71 & 92.0 \\
\hline V (cm/s) (95) & 15.55 & 19.90 & 16.37 & 110.84 \\
\hline
\end{tabular}

Fig. 4(a) and (b) shows the scatter plots of both the $u$ and $v$ components of the best case (the second image pair) and helps illustrate the values in Table II, which shows that, for the meridional $v$ components, the discrepancy between the LAC and GAC fields is larger at $30.43 \%$ than for the zonal $u$ components at $16.48 \%$. This is evident from Fig. 4(a) and (b), showing that the LAC and GAC $u$ components are a closer estimate of each other, looking at the $y$ intercept of the trendline. The $v$ components might be more closely correlated, which is indicated by the slightly higher determination coefficient, but the higher $y$ intercept of the trendline suggests a shifted origin, resulting in an overestimation of the LAC components by the GAC components. Fig. 4(a) and (b) shows 64 and 65 vector components, respectively, in which not all are visible as some of the dots are superimposed, indicating identical LAC/GAC value pairs for both $u$ and $v$.

The likely reason for the lower errors between the zonal $u$ LAC and GAC components compared to the meridional $v$ components for all three image pairs is that GAC data are computed from a sample averaging of the LAC data. Four out of every five samples along the scan line are used to compute one average value, and the data from only every third scan line are processed, thus giving a real resolution of $4.4 \times 1.1 \mathrm{~km}$. This procedure means that, in the meridional direction, the GAC data have gaps. Even though these are filled during the navigational interpolation algorithm, they still affect the feature tracking of the MCC, as it creates an uncertainty in the location of the original and final location of the feature. While the results of the second-image pair are encouraging, the two other cases do not demonstrate the same potential to retrieve current information from GAC images, as the LAC/GAC rms vector differences that should be low for accurate retrieval are too high and, in some cases, are actually higher than the LAC rms values, resulting in a $116 \%$ and $110 \%$ degradation of the $v$ component of the first and third image pairs, respectively.

As stated in Section III, the method precision also depends heavily on the accurate navigation of the images and on a precise attitude error correction. GAC images were attitude corrected using the correction parameters obtained for the LAC images, because it was not possible to correct these images independently with sufficient precision. This means that routine MCC processing of GAC images is problematic since it would involve the attitude error correction of the LAC images that would then be applied to the GAC images. Furthermore, current 


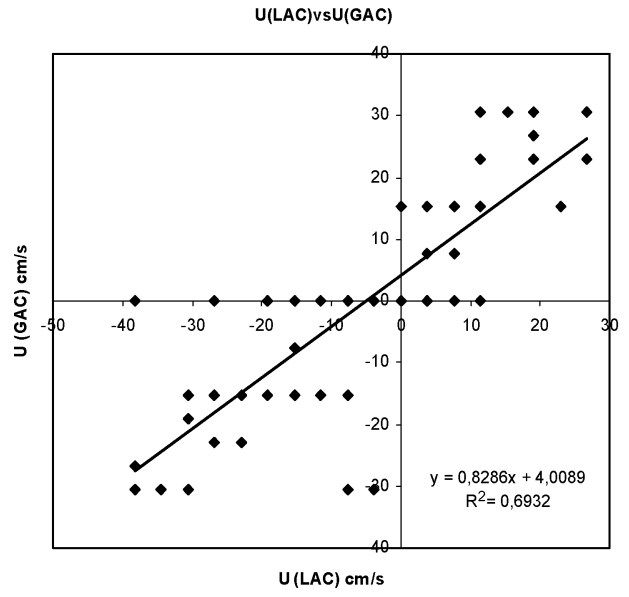

(a)

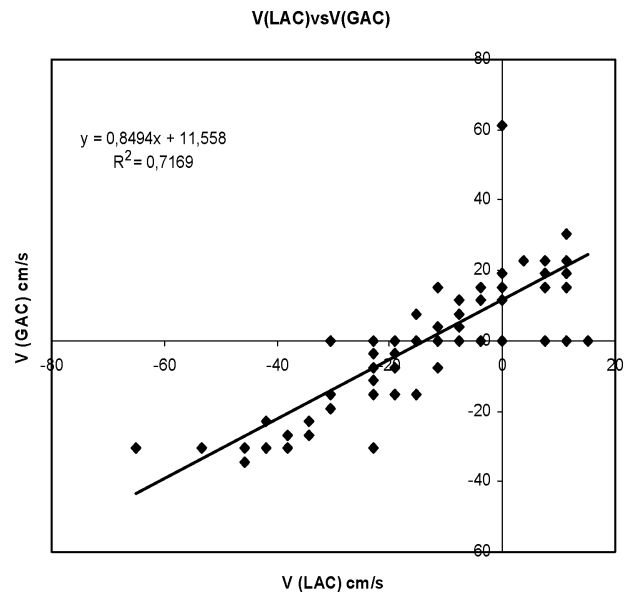

(b)

Fig. 4. (a) Scatterplot for the zonal-flow $u$ component. (b) Scatterplot for the meridional-flow $v$ component.

navigation and attitude error correction algorithms tend to rely on ground control points whose latitudes and longitudes are known to "anchor" the images to these points. This limits any accurately registered images of the coastal zones, i.e., the zones containing a landmass. The open ocean areas therefore tend to be less accurately registered and may introduce the spurious vectors if used in an MCC run.

\section{CONCLUSION}

We have shown that, in its current format, GAC data do not allow the retrieval of valid current information for all cases and, thus, on a routine basis. Averaging LAC data in a complete way to produce the "real" $4.4 \times 4.4 \mathrm{~km}$ GAC data may be a valid future step, as it should show whether the performance issues are related to the sampling of the field of vision. Other steps may include the use of larger template windows and also smoothing the final vector fields to reduce random noise. Streamfunctions may be fitted to the fields for smoothing the vector fields, but they also eliminate the ageostrophic flow components. An alternative to using the MCC method is the inversion of a heat-advection equation, as shown by Kelly [7], which may be less impacted by the more coarse GAC data. If it is demonstrated that the current vectors can be reliably retrieved from GAC data, it could be successfully applied to the sea-surface temperature fields from geostationary satellites that typically have 30-min time separations and may thus be easily composited to reduce cloud cover. Until it is shown that
GAC resolution images produce accurate vector fields, this is, however, not a viable objective.

\section{ACKNOWLEDGMENT}

The authors would like to thank W. Emery, D. Baldwin, D. Matthews and I. Crocker from the Colorado Center for Astrodynamical Research for the provision of and help with the various MCC routines.

\section{REFERENCES}

[1] M. M. Bowen, W. J. Emery, J. L. Wilkin, P. C. Tildesley, I. J. Barton, and R. Knewtson, "Extracting multiyear surface currents from sequential thermal imagery using the maximum cross-correlation technique," J. Atmos. Ocean. Technol., vol. 19, no. 10, pp. 1665-1676, 2002.

[2] S. Cote and A. R. L. Tatnall, "The Hopfield neural network as a tool for feature tracking and recognition from satellite sensor images," Int. J. Remote Sens., vol. 18, no. 4, pp. 871-885, Mar. 1997.

[3] W. J. Emery, C. Fowler, and C. A. Clayson, "Satellite-image-derived gulf stream currents compared with numerical model results," J. Atmos. Ocean. Technol., vol. 9, no. 3, pp. 286-304, 1992.

[4] W. J. Emery, A. C. Thomas, M. J. Collins, W. R. Crawford, and D. L. Mackas, "An objective method for computing advective surface velocities from sequential infrared satellite images," J. Geophys. Res., vol. 91, no. C11, pp. 12865-12 878, Nov. 1986.

[5] C. A. E. Garcia and I. S. Robinon, "Sea surface velocities in shallow seas extracted from sequential coastal zone color scanner satellite data," J. Geophys. Res., vol. 94, no. C9, pp. 12 681-12 691, Sep. 1989.

[6] R. J. Holyer and S. H. Peckinpaugh, "Edge detection applied to satellite imagery of the oceans," IEEE Trans. Geosci. Remote Sens., vol. 27, no. 1, pp. 46-56, Jan. 1989.

[7] K. A. Kelly, "An inverse model for near-surface velocity from infrared images," J. Phys. Oceanogr., vol. 19, no. 12, pp. 1845-1864, 1989. 